\title{
EL CRECIMIENTO ECONÓMICO PERUANO Y LA RESPONSABILIDAD SOCIAL DE LAS EMPRESAS
}

PERUVIAN ECONOMIC GROWTH AND CORPORATE SOCIAL RESPONSIBILITY

\author{
Julio Andrés Palomino Silva* \\ Docente Asociado de la Facultad de Ciencias Contables - UNMSM \\ [Recepción: Setiembre de 2011/ Conformidad: Octubre de 2011]
}

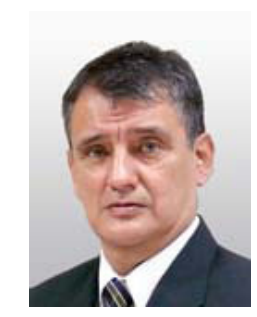

"No hay empresa exitosa en una sociedad fracasada así como ninguna sociedad será exitosa con empresas fracasadas"

Stephan Schmidheiny

\section{RESUMEN}

El rol del Sector Privado dentro de la sociedad, está cambiando frente a la nueva configuración de la economía global y local, por ello la actitud de renovarse y adecuarse a las necesidades y retos actuales.

El mercado, cada vez más exigente, en cuanto a la protección del entorno social y ambiental, hace necesario que las Empresas incorporen el ámbito de su responsabilidad social en sus políticas institucionales. Este nuevo enfoque de gestión empresarial, genera por una parte una corriente de consumo responsable y por otra, incentiva y apoya la inversión ética con responsabilidad de su entorno.

La mayoría de países han logrado obtener una tasa elevada de inversión local y atraer la inversión internacional justamente empleando este enfoque, que logra el escenario adecuado para la inversión.

En nuestro país, sin embargo, este nuevo rol social de las empresas se viene implementando a un nivel muy elemental, debido a que la responsabilidad social es vista como un gasto innecesario y no como una inversión, desmereciendo el hecho de que el aspecto social es determinante para la existencia y continuidad de la Empresa, coexistiendo ambas en estrecha relación, donde las transformaciones que ocurren en una afectan a la otra. Lograr vislumbrar esta relación condicional Empresa - responsabilidad social, determinar como es vista la responsabilidad empresarial en el marco del crecimiento económico nacional, como

\begin{abstract}
The Private Sector Role in society is changing before the new configuration of global and local economy, so the renewed attitude and meet the needs and challenges.

The market is increasingly demanding in terms of protecting the social and environmental context, requires that the companies incorporated within their social responsibility in their institutional policies. This new approach to corporate governance, on the one hand generates a stream of responsible consumption and the other, encourages and supports ethical investment responsibility for their environment.

Most countries have achieved a high rate of domestic investment and attract international investment just using this approach, which achieves the stage for investment.

In our country, however, this new social role of companies is being implemented at a very basic level, because social responsibility is seen as an unnecessary expense and not as an investment, desmereciendo the fact that the social aspect is determining the existence and continuity of the Company, both coexisting in close relationship, where the transformations that occur in one affect the other. Getting a glimpse of this relationship conditional Company - social responsibility, to determine how corporate responsibility is seen in the context of national economic growth, as set forth this new approach and help the company considers its social responsibility as an
\end{abstract}

* Magíster en Ciencias Contables y Empresariales- UNMSM, Docente de la Facultad de Ciencias Contables - UNMSMS, Docente Investigador de la Facultad de Ciencias Contables - UNMSM 
se establece este nuevo enfoque y ayudar a que la empresa considere su responsabilidad social como una inversión, es el objetivo de este artículo que encaja a la perfección con la Declaración del Milenio de Naciones Unidas, con el Programa de Doha para el Desarrollo y la Declaración de Johannesburgo cuyo objetivo es precisamente promover una mejor distribución de las funciones del Estado, la Empresa y la Sociedad Civil como actores en pos del desarrollo y la lucha contra la pobreza.

Palabras Clave: Crecimiento Económico, Empresa, Responsabilidad Social.

\section{INTRODUCCIÓN}

La Globalización económica, con la internacionalización del comercio, la práctica del libre mercado, de fronteras permeables, de cambios permanentes, con entornos de crisis, recesión y reconstrucción de sus economías, tienen poblaciones que paradójicamente al crecimiento económico no reciben los frutos de este; lo que acrecienta la necesidad de repotenciar el rol de actores estratégicos como El Estado, La Empresa y la misma Sociedad Civil.

El presente artículo se plantea determinar como es vista la responsabilidad social de las empresas en el marco del crecimiento económico nacional, como se establece este nuevo enfoque y ayudar a que la empresa considere su responsabilidad social como una inversión en el entorno que la circunscribe, teniendo como fundamento que es una inversión más para la empresa y que el aspecto social es determinante para su permanencia en la actual coyuntura.

La empresa dentro del marco de la teoría capitalista tiene como objetivo fundamental la producción de bienes y servicios, acorde con el pensamiento neoliberal que se propone incrementar al máximo las ganancias de esta. investment, the objective of this item that fits perfectly with the Millennium Declaration, United Nations, with the Doha Agenda for Development and the Johannesburg Declaration whose purpose is to promote a better distribution of state functions, Enterprise and Civil Society actors achieving development and poverty reduction.

Keywords: Economic Growth, Business, Social Responsibility.

Para la empresa como parte del sistema social y en la coyuntura que le ha tocado desenvolverse, es vital para asegurar su estabilidad y continuidad en el medio, cumplir con objetivos sociales, esto es, con su responsabilidad social.

Sin embargo, hasta hace poco se consideraba que los problemas sociales eran "todos" responsabilidad del Estado.

En la actualidad es bien sabido que el Estado no se da abasto para cubrir las necesidades de la población que presiona aún más al sector privado ha tomar parte de esa responsabilidad.

Lo aquí expresado no significa que la Empresa va a sustituir al Estado o que es ella quien debe resolver los problemas comunitarios y satisfacer las necesidades sociales, de ninguna manera, pero sí que debe tener un rol más activo y comprometido con su entorno social.

Este cambio también se hace extensivo al Estado, que tradicionalmente cumplía con un rol paternalista y asistencialista marcadamente subsidiario, el cual debe ser sustituido por un Estado promotor y regulador de la economía y que apunte a un crecimiento económico sostenible con equidad social.

Ahora bien, cual es el problema con este nuevo rol que debe asumir la Empresa, pues 
que para esta, la responsabilidad social significa un gasto innecesario y no es vista como una inversión a largo plazo.

Esta visión es la que le quita el espíritu empresarial a la responsabilidad social que deberían tener todas las empresas, claro está de acuerdo a su dimensión.

Nos queda claro que la Empresa debe centrar sus esfuerzos en producir bienes y servicios con la mayor eficacia y eficiencia para aumentar sus ganancias y que el Estado debe velar por la satisfacción de las necesidades y problemas sociales, pero ello no quita que ambos actores tengan una responsabilidad social que cumplir para con su entorno inmediato.

Nos toca demostrar entonces, que la nueva configuración escénica global condiciona el desarrollo social a la permanencia y estabilidad de la empresa, es decir que determina su existencia, porque el desarrollo social es visto como el marco de acción para la inversión privada, nacional e internacional; si la Empresa asume su responsabilidad social entonces mejorará las condiciones de vida de la sociedad en que se desenvuelve y que es su marco de desarrollo; la mayoría de países han logrado obtener una tasa elevada de inversión local y atraer la inversión internacional justamente empleando esta estrategia, asumiéndola con "responsabilidad" al crear un clima adecuado para la inversión.

Crear este marco de desarrollo social favorable para la inversión y que beneficia a la sociedad en su conjunto resalta el papel crucial de la Empresa dentro de la estrategia para el desarrollo del país.

Lograr una relación condicional entre Empresa y Responsabilidad Social es una necesidad para dar el siguiente paso dentro del avance de nuestra economía nacional.

Finalmente, la importancia de esta temática encaja a la perfección en la Declaración del Milenio de Naciones Unidas, con el Programa de Doha para el Desarrollo y la Declaración de Johannesburgo cuyo objetivo es promover una mejor distribución de funciones del Estado, la Empresa y la Sociedad Civil como actores en pos del desarrollo y la lucha contra la pobreza.

\section{EL ESCENARIO ECONÓMICO INTERNACIONAL}

En el escenario económico internacional observamos una tendencia que apunta a la recuperación de la actividad mundial a través de la confianza de empresas y consumidores en mayores y nuevas inversiones; lenta y débilmente vamos alejándonos del fantasma de la recesión con déficit, la abrupta desaceleración del consumo y el desempleo generados por la crisis de los Estados Unidos, lo que no implica que se hayan superado.

Considero que la crisis económica recesiva mundial está aún lejos de haber finalizado, por razones como el déficit fiscal que ha provocado que el Estado limite el gasto social a lo mínimo, a su vez el crédito es poco fluido lo que limita la reactivación del consumo manteniendo a gigantes sectores de consumidores con capacidad de gasto restringido, lo que a su vez impide la franca recuperación de la economía mundial; y el desempleo que es una bomba social que repentinamente puede cambiar el panorama de estabilidad y desarrollo que se necesita para lograr atraer a la inversión nacional y extranjera; pienso que ese es un tema obligado en las agendas de trabajo de todos los países, ya que es el medio biótico propicio para una nueva crisis, esta vez social, éste ha sido el costo social producto de la forma abrupta optada para salida de la crisis recesiva y que aún no nos ha pasado la totalidad de la factura como economía global.

Aún así, somos positivos al vislumbrar el panorama local. Nuestro país es uno de los 
que ha mostrado el crecimiento más alto de la región en base a su sector exportador, su inflación es la más baja de la región con sólo $2.0 \%$ y se ha convertido en uno de los países de destino de inversión más propicios del mundo.

Esta mejora de nuestra economía concretamente se ha visto reflejada en mayores exportaciones, recuperación del consumo, mayores inversiones, aumento de la recaudación, disminución de la salida de capitales, baja inflación, estímulos fiscales etc.; no obstante cabe ser cauteloso con la recuperación inicial de la economía mundial y local, a pesar de los indicios mostrados, debido a que no hay una tendencia clara porque el modelo económico mundial se está redefiniendo lo que implica la volatilidad en las economías.

\section{LAS NUEVAS REGLAS DE JUEGO}

La globalización es un proceso multidimensional derivado de los avances en la ciencia y la tecnología, que ha trastocado el escenario global y por ende el local; ante la complejidad de este proceso los roles económicos, políticos y sociales desempeñados tradicionalmente deben repensarse a la luz de este nuevo entorno.

Es una exigencia rediscutir el papel del Estado, la sociedad civil y el sector privado para impulsar el crecimiento, sólo realizando una reestructuración de los roles podremos encontrar el camino al desarrollo económico sostenible con equidad social y sustentabilidad en el uso de los recursos naturales; la economía no funciona sin capital natural, ni sin capital humano, son primordiales para el sistema económico, por lo que el sector privado les debe conceder la importancia debida.

Sin embargo, el sector industrial, eminentemente extractivo, muy pocas veces considera su interrelación con el entorno y evidentemente le causa un perjuicio por su falta de

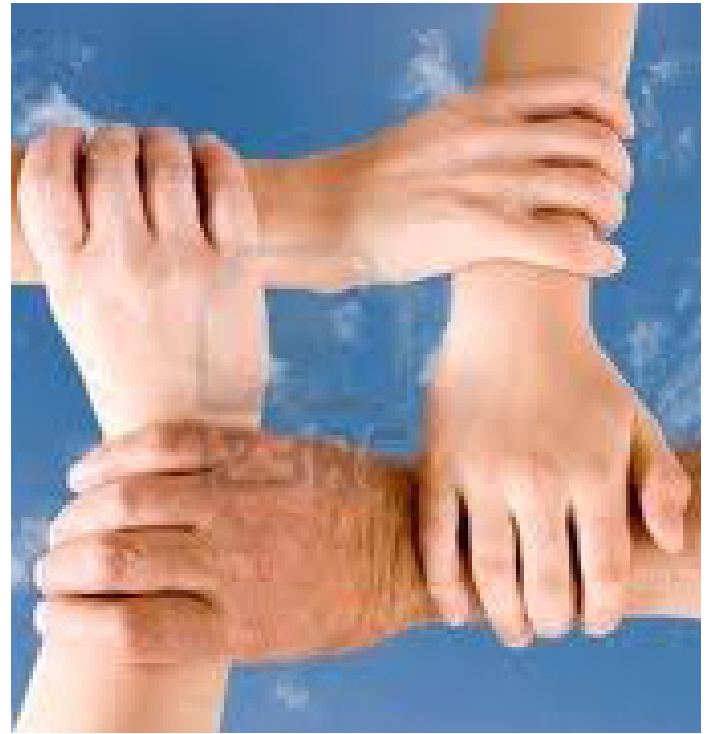

responsabilidad, situación que se revierte en su contra, porque el mercado es cada vez más exigente en cuanto al origen de los insumos de los productos, ello hace necesario que incorporen dentro de sus procesos productivos la gestión ambiental dentro del ámbito de su responsabilidad social, este nuevo enfoque de gestión empresarial, genera por una parte una corriente de consumo responsable y por otra, incentiva y apoya la inversión ética con responsabilidad de su entorno.

Es decir, es una forma ética de gestión que implica que la empresa tome conciencia de su rol en el desarrollo sostenible del país, asumiéndose como actor estratégico para este cambio.

Si el desarrollo económico del sector privado genera efectos adversos en su entorno, entonces que sentido tiene el desarrollo económico si éste va a ir en detrimento de su entorno inmediato - la sociedad, el medioambiente $-\mathrm{y}$ no va servir para satisfacer algunas necesidades de este; he ahí la necesidad de aplicar este nuevo enfoque, puesto que una solución efectiva para apalear esta situación es concientizar al sector privado, es decir la empresa, de la necesidad de que sea socialmente responsable, como está ocurriendo 
con otros países, donde la responsabilidad social es parte fundamental de sus políticas institucionales y agendas de trabajo.

Nuestro país no escapa a esta regla, somos parte de ese gran sistema económico imperante que condiciona no sólo nuestra economía, sino todo nuestro desarrollo como país, por lo que es momento de reasignar estos roles para equilibrar el peso de la responsabilidad social involucrando a la sociedad civil y al sector privado en especial, dentro del cual, la empresa juega un papel primordial al invertir en la construcción de un contexto de desarrollo social propicio para la inversión y que apuntará al desarrollo sostenible y con equidad social de nuestro país.

Es decir, las nuevas reglas de juego implican un nuevo pacto social donde la Empresa aprehenda que el aspecto social es el que determina la existencia y continuidad de la Empresa, coexistiendo en estrecha relación, donde las transformaciones que ocurren en una afectan a la otra.

Siendo los peruanos tan creativos, debemos encontrar nuevas maneras para interactuar como sociedad, acorde con las nuevas exigencias de competitividad donde la Empresa ocupe su sitial como actor central de nuestra economía y su rol activo dentro de la sociedad, velando tanto por su responsabilidad económica como social, con la visión de que su responsabilidad social es una inversión más para generar ganancias, ya que si es vista como un gasto entonces por principio rector estará yendo en contra de los intereses y del mismo espíritu empresarial; dado que ahora hablamos de un papel activo de la Empresa y no del papel pasivo que jugaba hasta hace poco, donde simplemente cumplía con una misión filantrópica de apoyo a la sociedad, como la reacción benéfica ante una desgracia motivada por una estrategia de marketing; este nuevo enfoque exige mucho más que eso como ya hemos visto.

Sobre el rol del Estado este debería ser el abanderado de este enfoque a través de las empresas públicas, además podría otorgar algunos estímulos institucionales y definitivamente debe constituirse en el eje generador de una cultura de responsabilidad social que encause al resto de actores sociales.

\section{HACIA UN CONTEXTO DE RESPONSABILIDAD SOCIAL EMPRESARIAL NACIONAL}

Aceptando el hecho de que la empresa como parte del sistema social tiene obligaciones y una de ellas es su responsabilidad social, la empresa está asumiendo nuevos roles de gestión y gerencia con un compromiso continuo de contribuir al desarrollo económico sostenible de nuestra sociedad.

La responsabilidad social no implica simplemente crear empleo y generar riqueza, implica promover el desarrollo del medio que rodea la empresa, viendo esto como una gestión ética del negocio que genera valor agregado a la misma, por lo que de ninguna manera es un costo, es una inversión de retorno a mediano y largo plazo

Para las empresas, la gestión bajo este esquema, no sólo les brindará mayores beneficios sino que las ayudará a elevar su competitividad, reducir riesgos y mejorar su reputación, de esta manera los beneficios son mutuos

Entendiendo la responsabilidad social como un complemento al desarrollo sostenible de la sociedad y para que sea entendida como tal - no solamente como una actividad aislada sino como un concepto completo - es necesario incorporarla como una herramienta estratégica transversal a todas las áreas de la empresa, así mismo, se deben establecer compromisos con clientes, accionistas, em- 
pleados y proveedores, pero también con toda la comunidad de la que forman parte.

En nuestro caso, en el Perú existe una voluntad de promover el ejercicio de la responsabilidad social, pero motivada básicamente por la presión externa y no por haber aprehendido todo lo que ello significa e implica, es decir, actúa condicionadamente como una estrategia de promoción y no por convicción, pues justamente la económica nacional actual se caracteriza por la apertura del mercado interno a la economía internacional, y ello trae consigo una serie de requerimientos $y$ exigencias para poder entrar a competir en ella.

El mercado internacional ante esta situación prefiere trabajar con empresas con buena reputación en cuanto a su responsabilidad social y castiga los pasivos ambientales y sociales en el valor de la acción, es por ello que las empresas compiten por desarrollar las mejores prácticas de gestión responsable, ya que mejora la credibilidad externa.

Pero en nuestro país la situación es más compleja. La responsabilidad social es una forma de gestión que implica la inclusión y participación de todos los grupos de interés: accionistas, inversionistas proveedores, consumidores, clientes, gobierno, sociedad, comunidad en general, medio ambiente, etc. que se encuentran alrededor de la empresa para lograr el desarrollo sostenible, y en nuestro caso no es así.

Entonces, ¿cuál es el papel de estos actores con respecto a la responsabilidad social, que hace el Estado, que hace la empresa y la misma comunidad en general, dado que nuestra sociedad se caracteriza por su tradición asistencialista?

En cuanto al Estado, su apoyo es total, en el discurso, pero en la practica es otra la situación, ya que no existe un respaldo con- creto a las actividades de la empresa en esa gestión, son muy pocas las empresas que han incorporado la responsabilidad social en la práctica real.

Sobre la comunidad, ésta reacciona esporádicamente, cuando se ven en extremo afectadas y resulta difícil negociar una vez dada la confrontación abierta. Tienen un papel pasivo, poco participativo.

Las empresas nacionales no son conscientes de que su actividad genera un impacto social y ambiental y sólo reaccionan cuando la población local donde operan las presiona, más no hacen nada por preveer esta situación, las pocas empresas que sí lo hacen la usan como una estrategia para el logro de la viabilidad de sus proyectos.

Finalicemos entonces, determinando que la responsabilidad social empresarial es una metodología de gestión que en nuestro país debe partir por un cambio de actitud que nos encamine al desarrollo sostenible que proteja el medio ambiente y a las comunidades sin descuidar sus intereses económicos.

\section{CONCLUSIONES}

1. La responsabilidad social empresarial es una forma ética de gestión, mediante la cual las empresas asumen un rol activo como agente de cambio y se comprometen a participar en el desarrollo sostenible del país mejorando su entorno.

2. La responsabilidad social empresarial es una estrategia más de la empresa, es una inversión más para generar ganancias porque se vuelven más competitivas y pueden obtener mayores ganancias dado que en el contexto actual la dimensión social es determinante al momento de realizar una elección. 
3. En este proceso se deben involucrar además todos los grupos de interés para lograr el desarrollo sostenible: accionistas, inversionistas proveedores, consumidores, clientes, gobierno, sociedad, comunidad en general, medio ambiente, etc.

4. En nuestro país, la participación del Estado, las empresas y la comunidad en general, en cuanto al tema de la responsabilidad social es insuficiente para mejorar las condiciones de vida de las comunidades donde operan, y mucho menos como para lograr el tan anhelado desarrollo sostenible.

5. Incidimos en que la responsabilidad social debe ser asumida corporativamente, afianzando alianzas entre los grupos de interés involucrados que deben partir de un cambio de actitud para convertirse en instrumentos facilitadores de un adecuado proceso de desarrollo equitativo y sustentable en nuestro país.

6. La responsabilidad social empresarial abre importantes oportunidades de inversión, financiamiento y asistencia técnica estratégica, las perspectivas en este ámbito son ambiciosas, pero requieren del apoyo concertado de los grupos involucrados.

\section{BIBLIOGRAFÍA}

1. Conferencia Internacional del Trabajo. 96a Reunión. 2007 La promoción de Empresas Sostenibles. OIT.

2. Córdoba Largo, Alejandro. 2007 El corazón de las empresas. Responsabilidad Social Corporativa y Conciliación de la vida profesional y personal. ESIC Editorial.
3. Cuba Baiocchi, Carmen Marina.

Responsabilidad Social Empresarial. www.urp.edu.pe/urp/modules . $(15 / 10 / 2011)$

4. Curso "Responsabilidad Social Empresarial y Gestión de las Relaciones Comunitarias en la Industria Minera”.

2008 Síntesis del diagnóstico elaborado por los asistentes a la primera sesión. Lima Perú.

5. Martínez Herrera, Horacio. 2005 El marco ético de la Responsabilidad Social Empresarial. Editorial Pontificia Universidad Javeriana.

6. Navarro García, Fernando. 2008 Responsabilidad Social Corporativa. Teoría y Práctica. ESIC Editorial.

7. Paladino, M., Alvarez Tejeiro, C., Willi, Alberto \& otros.

2004. La responsabilidad de la empresa en la sociedad. Buenos Aires: Ariel Sociedad Anónima.

8. Perdiguero, Tomás G. \& García Reche, Andrés (Editores). 2005 La Responsabilidad Social de las Empresas y los nuevos desafíos de la Gestión Empresarial. PUV.

9. Raich, Mario \& Dolan Simon L. 2009 “Más Allá. Empresa y Sociedad en un mundo en transformación." Profit Editorial.

10. Restrepo González, Ramiro. 2009 Responsabilidad Social. Nuevas Teorías, Nuevas Prácticas.

11. labuenaempresa.wordpress.com/.../ nuevo-libro-responsabilidad-social. 22/11/2011). 